\title{
The origin of organic emission in NGC 2071^
}

\author{
T. A. van Kempen ${ }^{1}$, C. $\mathrm{M}^{\mathrm{c}} \mathrm{Coey}^{2}$, S. Tisi ${ }^{2}$, D. Johnstone ${ }^{3,4,5}$, and M. Fich ${ }^{2}$ \\ 1 Leiden Observatory, Leiden University, Niels Bohrweg 2, 2333 CA Leiden, The Netherlands \\ e-mail: kempen@strw. leidenuniv.nl \\ 2 Department of Physics and Astronomy, University of Waterloo, Waterloo, Ontario, N2L 3G1, Canada \\ 3 Joint Astronomy Center, 660 North A'ohoku Place, University Park, Hilo, HI 96720, USA \\ ${ }^{4}$ National Research Council Canada, Herzberg Institute of Astrophysics, 5071 West Saanich Rd, Victoria, BC, V9E 2E7, Canada \\ 5 Department of Physics \& Astronomy, University of Victoria, Victoria, BC, V8P 1A1, Canada
}

Received 17 March 2014 / Accepted 8 July 2014

\section{ABSTRACT}

\begin{abstract}
Context. The physical origin behind organic emission lines in embedded low-mass star formation has been fiercely debated over the last two decades. A multitude of scenarios have been proposed, from a hot corino to PDRs on cavity walls to shock excitation.

Aims. The aim of this paper is to determine the location and the corresponding physical conditions of the gas responsible for organics emission lines. The outflows around the small protocluster NGC 2071 are an ideal testbed that can be used to differentiate between various scenarios.

Methods. Using Herschel-HIFI and the Submillimeter Array, observations of $\mathrm{CH}_{3} \mathrm{OH}, \mathrm{H}_{2} \mathrm{CO}$, and $\mathrm{CH}_{3} \mathrm{CN}$ emission lines over a wide range of excitation energies were obtained. Comparisons to a grid of radiative transfer models provide constraints on the physical conditions. Comparison to $\mathrm{H}_{2} \mathrm{O}$ line shape is able to trace gas-phase synthesis versus a sputtered origin.

Results. Emission of organics originates in three separate spots: the continuum sources IRS 1 ("B") and IRS 3 ("A") and a new outflow position ("F"). Densities are above $10^{7} \mathrm{~cm}^{-3}$ and temperatures between $100 \mathrm{~K}$ and $200 \mathrm{~K}$. $\mathrm{CH}_{3} \mathrm{OH}$ emission observed with HIFI originates in all three regions and cannot be associated with a single region. Very little organic emission originates outside of these regions.

Conclusions. Although the three regions are small $(<1500 \mathrm{AU})$, gas-phase organics likely originate from sputtering of ices as a result of outflow activity. The derived high densities $\left(>10^{7} \mathrm{~cm}^{-3}\right)$ are likely a requirement for organic molecules to survive from being immediately destroyed by shock products after evaporation. The lack of spatially extended emission confirms that organic molecules cannot (re-)form through gas-phase synthesis, as opposed to $\mathrm{H}_{2} \mathrm{O}$, which shows strong line wing emission. The lack of $\mathrm{CH}_{3} \mathrm{CN}$ emission at " $\mathrm{F}$ " is evidence for a different history of ice processing because of the absence of a protostar at that location and recent ice mantle evaporation.
\end{abstract}

Key words. stars: formation - submillimeter: ISM - stars: protostars - circumstellar matter

\section{Introduction}

Strong emission lines of hydrogenated carbon-bearing species are a common feature associated with the early stages of star formation. Embedded high-mass protostars possess a so-called hot core component (Kurtz et al. 2000), from which large quantities of emission lines from organic molecules have been detected (e.g., Comito et al. 2005). This spherically symmetric region around the massive protostar is powered solely by luminosities from the newly ignited protostar and its accretion flow.

Similar emission features of organic compounds have been detected around low-mass protostars (van Dishoeck et al. 1995; Cazaux et al. 2003; Bottinelli et al. 2004a; Bisschop et al. 2008; Caux et al. 2011), including simple sugars (Jørgensen et al. 2012). Even though most emission lines of the organics clearly originate in the inner few hundred $\mathrm{AU}$, their physical origin is fiercely debated. In one scenario, emission originates in a spherically symmetric component around the protostar similar

* This paper uses Herschel observations. Herschel is an ESA space observatory with science instruments provided by Europeanled Principal Investigator consortia and with important participation from NASA. This paper also makes use of SMA observations. The Submillimeter Array is a joint project between the Smithsonian Astrophysical Observatory and the Academia Sinica Institute of A\&A and is funded by the Smithsonian Institution and the Academia Sinica. to the hot core, referred to as the "hot corino" (Ceccarelli et al. 2007). In some sources the molecular emission does not coincide with the positions where the continuum peaks and the protostar is believed to be located (e.g., Chandler et al. 2005). Observed line shapes, in particular the line wings, do not support a spherical model. Furthermore, SED and envelope modeling of lowmass protostars show that although central warm regions are possible (Shirley et al. 2002), typical sizes and column densities of the protostellar envelope are much larger and higher than predicted. Other sources of energy are required to explain the observations.

Abundances of molecules such as methanol and formaldehyde $\left(\mathrm{CH}_{3} \mathrm{OH}\right.$ and $\left.\mathrm{H}_{2} \mathrm{CO}\right)$ are factors of $\sim 30-100$ higher in protostellar environments than in dark clouds (Takakuwa et al. 1998), where non-thermal desorption dominates (Öberg et al. 2009). From examination of the physical and chemical conditions, release of icy grain mantles must play a crucial role (van der Tak et al. 2000; Maret et al. 2005). It is poorly understood in which situations thermal desorption of water $\left(\mathrm{H}_{2} \mathrm{O}\right)$ (Sandford \& Allamandola 1993) is dominant, when grain-grain collisions that shatter ice mantles (Jones et al. 1996) are required, or when sputtering by energetic particles produced in shocks play a large role (Flower \& Pineau Des Forêts 2010). Whatever the method, $\mathrm{CH}_{3} \mathrm{OH}$ is a pure grain mantle product. 
Bottinelli et al. (2004b) and Bisschop et al. (2008) showed the importance of resolved interferometric observations of organic emission. By resolving components and their conditions, differences in the formation of organics can be uncovered. Abundances of some species cannot be produced by grain surface hydrogenation alone. A combination of grain surface reactions and gas phase formation and/or destruction is often required.

Shock desorption has been invoked to account for observed offsets of organic emission when visibly associated with outflowing material (e.g., Buckle \& Fuller 2002). Shocks and their associated cavities also allow for additional energy injections not possible in spherical models. Warm gas on the surface of outflow cavity walls has been invoked to explain observed CO $J=6-5$ emission (Spaans et al. 1995; van Kempen et al. 2009). There is no reason why this cannot be a physical driver for emission of organics, following the relatively high gas temperature of this component (Visser et al. 2012). An important constraint to the conditions is the amount of $\mathrm{CH}_{3} \mathrm{OH}$ destroyed, either through dissociative desorption during the sputtering or reactions with $\mathrm{H}$ in the shock. Recently, Suutarinen et al. (2014) found that comparison of $\mathrm{CH}_{3} \mathrm{OH}$ with $\mathrm{H}_{2} \mathrm{O}$ can probe the conditions of irradiated shocks.

Most likely, the various origins coexist. This is best shown by the ongoing work in intermediate mass protocluster OMC 2 FIRS 4 (Kama et al. 2010, 2013; López-Sepulcre et al. 2013). The small scales show more than four components, while the observed methanol emission cannot be tied to one component, even though $>100$ lines have been detected. Multiple components and origins are required.

The tightly packed proto-cluster NGC 2071, located at 422 pc (Carrasco-González et al. 2012; van Kempen et al. 2012) produces one of the most powerful outflows within $500 \mathrm{pc}$ (Scoville et al. 1986; Butner et al. 1990). In combination with the relatively low stellar masses of all three protostars and a cleaner environment, it is a better candidate with which to differentiate between outflow driven scenarios and hot-core like origins or quantify the relative contributions of both. It is rich in molecular emission (Johnstone et al. 2003), at times showing two Gaussian components of different widths.

In this paper, we present new results using observations of emission lines of three organic compounds: methanol $\left(\mathrm{CH}_{3} \mathrm{OH}\right)$, formaldehyde $\left(\mathrm{H}_{2} \mathrm{CO}\right)$, and methyl cyanide $\left(\mathrm{CH}_{3} \mathrm{CN}\right)$. These three organic compounds are commonly used as tracers for chemical activity in nearby star forming regions (e.g., Jørgensen et al. 2011; Kama et al. 2013). Observations were obtained with ESA Herschel Space Observatory (Pilbratt et al. 2010) using the Heterodyne Instrument for the Far-Infrared (HIFI; de Graauw et al. 2010), and the Submillimeter Array (SMA). The aim of this paper is to determine the location, excitation, and physical conditions of the region(s) responsible for the organic emission in NGC 2071 and differentiate between a hot corino scenario or one related to the outflowing gas. Section 2 presents the observations, and subsequent results are listed in Sect. 3. The analysis is done in Sect. 4. The origin scenario is discussed in Sect. 5, with conclusions summarized in Sect. 6.

\section{Observations}

Spectral line observations of HIFI (de Graauw et al. 2010) and the SMA were inspected for methanol $\left(\mathrm{CH}_{3} \mathrm{OH}\right)$, formaldehyde $\left(\mathrm{H}_{2} \mathrm{CO}\right)$ and/or methyl cyanide $\left(\mathrm{CH}_{3} \mathrm{CN}\right)$ emission lines. To aid the analysis into the conditions that set the chemistry the 987

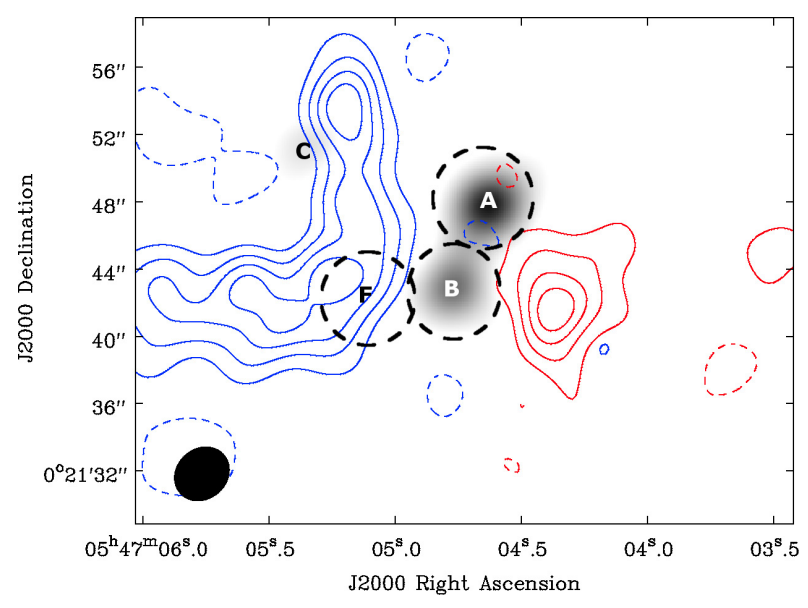

Fig. 1. Integrated line wings of ${ }^{13} \mathrm{CO}$ emission (blue and red) around NGC 2071 obtained with the SMA. It is overplotted on the $1.3 \mathrm{~mm}$ continuum emission (grayscale) in steps of 3, 6, 9, $12 \sigma$ (see van Kempen et al. 2012). Locations of continuum sources (A, B, and C) are labeled. The beam size of $3.4^{\prime \prime} \times 2.9^{\prime \prime}$ is shown in a black ellipse in the lower left. Dashed circles indicate the regions integrated for organic emission, including region $\mathrm{F}$ (see text).

Table 1. Observational parameters.

\begin{tabular}{|c|c|c|c|c|c|}
\hline \multicolumn{6}{|c|}{ HIFI } \\
\hline Setting & $\begin{array}{r}\text { Freq. }^{1} \\
\text { GHz }\end{array}$ & Band & $\theta_{\mathrm{mb}}$ & ObsId. & $\begin{array}{r}\mathrm{rms}^{2} \\
\mathrm{mK}\end{array}$ \\
\hline 1 & 547.676 & $1 \mathrm{a}$ & 38.0 & 1342194490 & 5 \\
\hline 2 & 556.936 & $1 \mathrm{a}$ & 38.0 & 1342205274 & 15 \\
\hline 3 & 752.033 & $2 b$ & 28.0 & 1342194682 & 19 \\
\hline 4 & 987.927 & $4 a$ & 21.5 & 1342204503 & 25 \\
\hline 5 & 1095.67 & $4 \mathrm{~b}$ & 19.0 & 1342227395 & 17 \\
\hline 6 & 1113.343 & $4 \mathrm{~b}$ & 19.0 & 1342194790 & 18 \\
\hline 7 & 1153.127 & $5 \mathrm{a}$ & 18.5 & 1342206128 & 93 \\
\hline \multicolumn{6}{|c|}{ SMA } \\
\hline \multirow{2}{*}{\multicolumn{3}{|c|}{$\begin{array}{c}\text { Bandwidth } \\
\text { Center frequencies }\end{array}$}} & \multirow{2}{*}{\multicolumn{3}{|c|}{$\begin{array}{c}2 \times 4 \mathrm{GHz} \\
2305 \mathrm{nd} 2190 \mathrm{GHz}\end{array}$}} \\
\hline & & & & & \\
\hline \multicolumn{3}{|c|}{ Configuration } & \multicolumn{3}{|c|}{$\begin{array}{l}250.5 \text { and } 219.0 \text { Unz } \\
\text { SMA Compact }\end{array}$} \\
\hline \multicolumn{3}{|c|}{ Beam size } & \multicolumn{3}{|c|}{$3.4^{\prime \prime} \times 2.9^{\prime \prime}$} \\
\hline \multicolumn{3}{|c|}{ Observation date } & \multicolumn{3}{|c|}{ January 13th 2010} \\
\hline
\end{tabular}

Notes. (1) Center frequency of the HIFI band. (2) Measured in $0.5 \mathrm{~km} \mathrm{~s}^{-1}$ bins.

and $752 \mathrm{GHz} \mathrm{H}_{2} \mathrm{O}$ lines are included in this paper (Suutarinen et al. 2014).

SMA observations of the line wings of ${ }^{13} \mathrm{CO} J=2-1$ (see Fig. 1) are used as a reference for the location of the outflowing gas. Table 1 lists the observational settings of both the SMA and Herschel-HIFI observations. Data reduction was performed using a combination of the following software tools: the MIR package for IDL, MIRIAD, HIPE ${ }^{1}$ (Ott 2010) and CLASS in GILDAS $^{2}$.

1 HCSS/HSpot/HIPE is a joint development (are joint developments) by the Herschel Science Ground Segment Consortium, consisting of ESA, the NASA Herschel Science Center, and the HIFI, PACS and SPIRE consortia.

2 GILDAS is a software package developed by IRAM to reduce and analyze astronomical data; http://www. iram.fr/IRAMFR/GILDAS 


\subsection{HIFI}

HIFI $^{3}$ observations of NGC 2071 were carried out within the scope of the WISH Key program (van Dishoeck et al. 2011), targeting rotational emission of $\mathrm{H}_{2} \mathrm{O}$. All pointings were centered at a position with a Right Ascension of $05^{\mathrm{h}} 47^{\mathrm{m}} 04.4^{\mathrm{s}}$ and a Declination of $00^{\mathrm{d}} 21^{\mathrm{m}} 49^{\mathrm{s}}$. Except the setting targeting the ground-state $\mathrm{H}_{2} \mathrm{O}$ line at $557 \mathrm{GHz}$ (setting 2), all observations were done using DBS Fast Chop modes (see Table 1 for the observed HIFI bands and Observations Identification Numbers). After delivery, data were reprocessed with the default pipeline in HIPE 7.1 (Ott 2010) and calibration version HIFI_CAL_6_0. Further data reduction was done using HIPE 8.1. H and V spectra were averaged together prior to converting the data to the velocity scale. The WBS spectrometer provided a $4 \mathrm{GHz}$ bandwidth with a $1.1 \mathrm{MHz}$ frequency resolution $\left(0.7\right.$ to $\left.0.4 \mathrm{~km} \mathrm{~s}^{1}\right)$. The beam of HIFI ranges from 18 to $41^{\prime \prime}$ much larger than the structure seen in van Kempen et al. (2012; see Table 1 and Fig. 1). Relative calibration errors are $15 \%$ for all Bands except the one in Band 5, which is $20 \%$.

\subsection{SMA}

The SMA observed NGC 2071 in compact configuration on January 3rd 2010 using a bandwidth of $4 \mathrm{GHz}$ centered at $230.538 \mathrm{GHz}$ and $4 \mathrm{GHz}$ centered at $219.634 \mathrm{GHz}$ and a spectral resolution of $0.3 \mathrm{~km} \mathrm{~s}^{-14}$. The phase center was located at a Right Ascension of $05^{\mathrm{h}} 47^{\mathrm{m}} 04.7^{\mathrm{s}}$ and a Declination of $+00^{\mathrm{d}} 21^{\mathrm{m}} 44.0^{\mathrm{s}}$. Continuum results were published in van Kempen et al. (2012). With a spatial resolution corresponding to $\sim 1300 \mathrm{AU}\left(3.4^{\prime \prime} \times 2.9^{\prime \prime}\right)$, the full extent of the flow is spatially resolved, as are the separations between most of the individual protostars (van Kempen et al. 2012). The noise levels were $4 \mathrm{mJy}^{\mathrm{beam}}{ }^{-1}$ for the continuum and $0.08 \mathrm{Jy}^{\text {beam }}{ }^{-1}$ per $0.8 \mathrm{~km} \mathrm{~s}^{-1}$ bin. this corresponds to $\sim 0.4 \mathrm{~K}$ in the $0.8 \mathrm{~km} \mathrm{~s}^{-1}$ bins. Calibration uncertainty in the lines was derived to be $15 \%$ after the model fitting of Uranus. We will follow the notation from van Kempen et al. (2012), in which the source IRS 3 is labeled as "A", IRS 1 as "B" and IRS 5 as "C". We note that the phase center is located in between " $A$ " and "B". As a result of the placement of correlator chunks, which optimized coverage for the CO isotopologues and continuum, several instrumental artifacts needed to be flagged at semi-regular intervals. The only one affecting emission lines of $\mathrm{H}_{2} \mathrm{CO}, \mathrm{CH}_{3} \mathrm{OH}$ or $\mathrm{CH}_{3} \mathrm{CN}$ appears between the $K=1$ and 2 transitions of the $J=12-11$ ladder of $\mathrm{CH}_{3} \mathrm{CN}$. The artificial feature is 8 channels wide with a regular increase per channel. Spectra were corrected by fitting and

\footnotetext{
3 HIFI has been designed and built by a consortium of institutes and university departments from across Europe, Canada and the United States under the leadership of SRON Netherlands Institute for Space Research, Groningen, The Netherlands and with major contributions from Germany, France and the US. Consortium members are: Canada: CSA, U.Waterloo; France: CESR, LAB, LERMA, IRAM; Germany: KOSMA, MPIfR, MPS; Ireland, NUI Maynooth; Italy: ASI, IFSI-INAF, Osservatorio Astrofisico di Arcetri- INAF; Netherlands: SRON, TUD; Poland: CAMK, CBK; Spain: Observatorio Astronómico Nacional (IGN), Centro de Astrobiología (CSIC-INTA). Sweden: Chalmers University of Technology - MC2, RSS \& GARD; Onsala Space Observatory; Swedish National Space Board, Stockholm University - Stockholm Observatory; Switzerland: ETH Zurich, FHNW; USA: Caltech, JPL, NHSC.

4 The calibrated data is available through the Radio Telescope Data Center hosted by the Smithsonian Astrophysical Observatory http:// www. cfa.harvard. edu/rtdc/
}

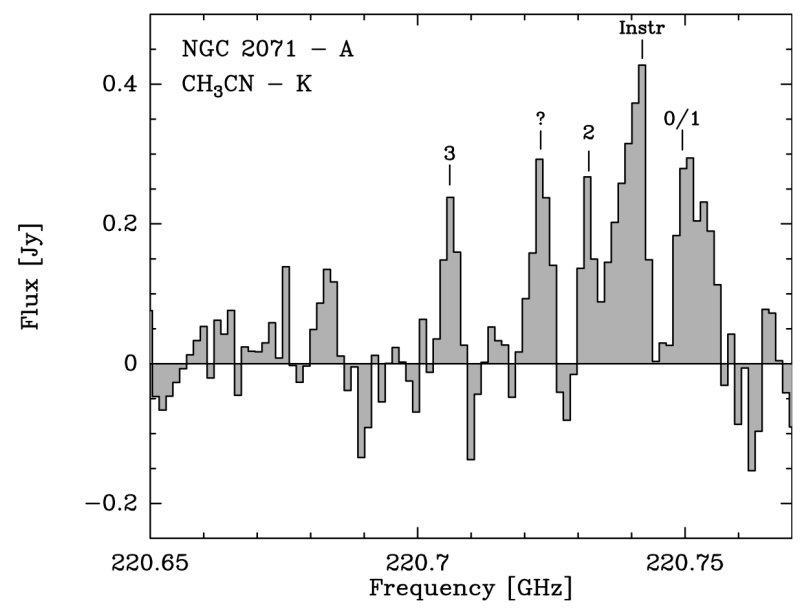

Fig. 2. $\mathrm{CH}_{3} \mathrm{CN} K=12-11$ ladder between 220.65 and $220.8 \mathrm{GHz}$ observed by the SMA at the position of region "A". The instrumental feature between the 2 and $0 / 1$ blended transitions is labeled with "Instr".

subtracting a step-function to these channels. Figure 2 presents the uncorrected $\mathrm{CH}_{3} \mathrm{CN}$ spectrum for source " $\mathrm{A}$ ".

\section{Results}

\subsection{HIFI}

In the HIFI bands, a total of $24 \mathrm{CH}_{3} \mathrm{OH}$ emission lines were identified, but no $\mathrm{CH}_{3} \mathrm{CN}$ or $\mathrm{H}_{2} \mathrm{CO}$ lines were seen. The $\mathrm{H}_{2} \mathrm{O}$ lines are both very clearly detected with very broad line wings $\left(>20 \mathrm{~km} \mathrm{~s}^{-1}\right.$, see Fig. 4). The rms noise levels can be found in Table 1. More details on water emission will be in a forthcoming paper ( $\mathrm{M}^{\mathrm{c}}$ Coey \& van Kempen, in prep.). Quantum numbers, rest frequencies, energy levels and velocity integrated flux densities of the detected transitions are listed in Table 2 . We note that no lines were found in Band 5, which has a higher calibration uncertainty than the other Bands. As such the uncertainty for all lines in Table 2 is assumed to be $15 \%$. Rest frequencies of all detections were checked against the molecular line survey of Kama et al. (2013). One feature was removed as questionable. Energy levels range from 75 to 500 Kelvin. The bulk of the lines are observed around frequencies of $766 \mathrm{GHz}$, within the $K_{\text {up }}$ to $K_{1}=-3$ to -2 ladder (See Fig. 3). All detected lines have $K_{\text {up }}=\leq|3|$. Lines are consistently $\approx 6 \mathrm{~km} \mathrm{~s}^{-1}$ wide. No evidence was found for an increase in line width as a function of energy level, as suggested by Kama et al. (2010). A very small shift in in velocity with respect to the source velocity is at times observed, but this is more likely to be an artifact of the methanol lines not being in the center of the HIFI bandpass and not a physical effect.

\subsection{SMA}

Table 3 lists the detected $\mathrm{CH}_{3} \mathrm{OH}, \mathrm{H}_{2} \mathrm{CO}$ and $\mathrm{CH}_{3} \mathrm{CN}$ transitions with quantum numbers, rest frequencies, energy levels and integrated flux densities. For $\mathrm{CH}_{3} \mathrm{OH}$, only transitions with $K_{\text {up }} \leq|2|$ are seen, with one line $K_{\text {up }}=0$. For $\mathrm{CH}_{3} \mathrm{CN}$, all detections are part of the $K=12-11$ ladder around $220.7 \mathrm{GHz}$. All detected lines have line widths ranging between 5.9 to $8.0 \mathrm{~km} \mathrm{~s}^{-1}$ wide, similar to the line widths of $\mathrm{CH}_{3} \mathrm{OH}$ detected by HIFI. Emission is spatially resolved into three positions for most lines (see Fig. 5). Integrated flux densities are given for the three positions, labeled "A", "B" and "F". The "F" position is a 


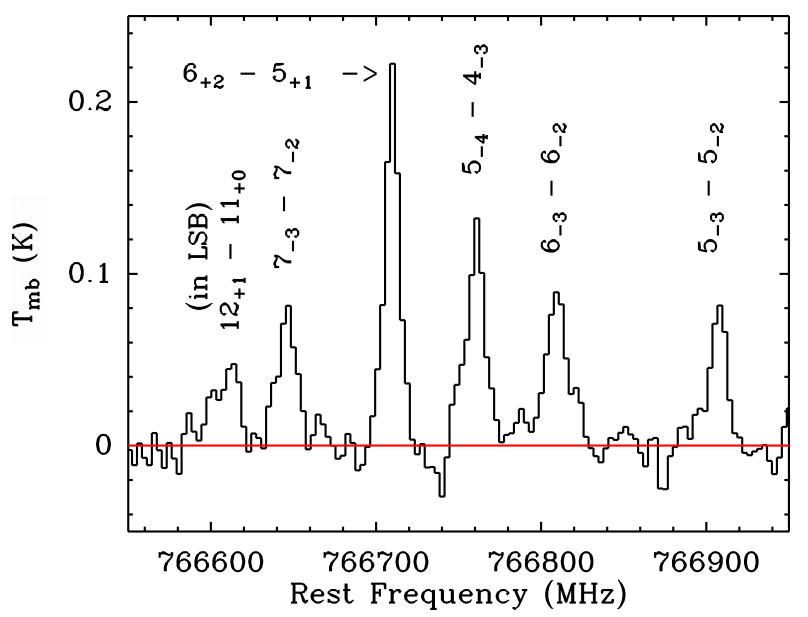

Fig. 3. Detected $\mathrm{CH}_{3} \mathrm{OH}$ lines observed with HIFI in $400 \mathrm{MHz}$ centered at $766.75 \mathrm{GHz}$. We note that the $12_{+1}-1_{+0}$ line is from the lower sideband, with a rest frequency of $751.55 \mathrm{GHz}$. Channels are resampled to a width of $0.75 \mathrm{~km} \mathrm{~s}^{-1}$. The baseline is shown as a horizontal red line. At this frequency, the beam of HIFI is $26^{\prime \prime}$.

Table 2. Emission lines detected with HIFI.

\begin{tabular}{lcccc}
\hline \hline Transition & Type & $\begin{array}{c}\text { Freq. } \\
{[\mathrm{GHz}]}\end{array}$ & $\begin{array}{c}E_{\text {up }} \\
{[\mathrm{K}]}\end{array}$ & $\begin{array}{c}\int T_{\mathrm{MB}} \mathrm{d} V \\
{\left[\mathrm{~K} \mathrm{~km} \mathrm{~s}^{-1}\right]}\end{array}$ \\
\hline \multicolumn{5}{c}{$\mathrm{CH}_{3} \mathrm{OH}$} \\
\hline $3_{-3}-3_{-2}$ & $\mathrm{E}$ & 766.98 & 76.64 & 1.03 \\
$4_{-3}-4_{-2}$ & $\mathrm{E}$ & 766.96 & 85.92 & 1.03 \\
$6_{+2}-5_{+1}$ & $\mathrm{~A}$ & 766.71 & 86.46 & 0.90 \\
$5_{-3}-5_{-2}$ & $\mathrm{E}$ & 766.91 & 97.53 & 0.41 \\
$8_{+0}-7_{+1}$ & $\mathrm{E}$ & 543.08 & 96.61 & 0.75 \\
$6_{-3}-6_{-2}$ & $\mathrm{E}$ & 766.81 & 111.46 & 0.56 \\
$5_{-4}-4_{-3}$ & $\mathrm{E}$ & 766.76 & 122.72 & 0.66 \\
$7_{-3}-7_{-2}$ & $\mathrm{E}$ & 766.65 & 127.71 & 0.39 \\
$10_{+1}-9_{+0}$ & $\mathrm{~A}$ & 763.95 & 141.08 & 1.19 \\
$7_{+4}-6_{+3}$ & $\mathrm{~A}$ & 974.87 & 145.33 & 0.88 \\
$8_{-3}-8_{-2}$ & $\mathrm{E}$ & 766.40 & 146.28 & 0.33 \\
$10_{+2}-9_{+1}$ & $\mathrm{~A}$ & 986.10 & 165.40 & 0.38 \\
$9_{-3}-9_{-2}$ & $\mathrm{~A}$ & 766.08 & 167.16 & $<0.30$ \\
$11_{+1}-10_{+1}$ & $\mathrm{~A}$ & 536.19 & 169.01 & 0.50 \\
$11_{+2}-10_{+1}$ & $\mathrm{E}$ & 558.34 & 175.15 & 0.40 \\
$10_{-3}-10_{-2}$ & $\mathrm{E}$ & 765.51 & 190.37 & 0.29 \\
$12_{+1}-11_{+0}$ & $\mathrm{E}$ & 751.55 & 202.12 & 0.27 \\
$11_{-3}-11_{-2}$ & $\mathrm{E}$ & 764.81 & 215.90 & 0.26 \\
$12_{+3}-11_{+2}$ & $\mathrm{E}$ & 1109.58 & 228.78 & 0.33 \\
$15_{+2}-14_{+1}$ & $\mathrm{E}$ & 754.22 & 300.98 & 0.36 \\
$18_{+1}-17_{+0}$ & $\mathrm{~A}$ & 1105.94 & 407.62 & 0.23 \\
$23_{+1}-22_{-2}$ & $\mathrm{~A}$ & 977.37 & 427.10 & 0.24 \\
$20_{+0}-19_{-1}$ & $\mathrm{~A}$ & 1095.06 & 497.93 & 0.23 \\
\hline \multicolumn{5}{c}{$\mathrm{H}_{2} \mathrm{O}$} \\
\hline $2_{02}-1_{11}$ & Para & 987.927 & 100.8 & 88.48 \\
$2_{11}-2_{02}$ & Para & 752.033 & 136.9 & 46.62 \\
\hline
\end{tabular}

Notes. The average uncertainty on the integrated intensity is estimated at $15 \%$ (see text).

position $4^{\prime \prime}$ west of B, with no continuum nor infra-red emission peak (van Kempen et al. 2012). Figure 1 shows " $F$ " to be a position associated with entrained material. No velocity shifts were found between the three different positions.

The observed spatial distributions were fitted with Gaussian profiles with the goal to determine whether or not emission was spatially resolved. Spatial scales $>1.5 \times$ the beam size along at least one axis were required. It was found that only $\mathrm{H}_{2} \mathrm{CO}$ emission from " $\mathrm{F}$ " and " $\mathrm{B}$ " is clearly spatially resolved. It is unresolved for "A". $\mathrm{CH}_{3} \mathrm{OH}$ emission is predominantly unresolved

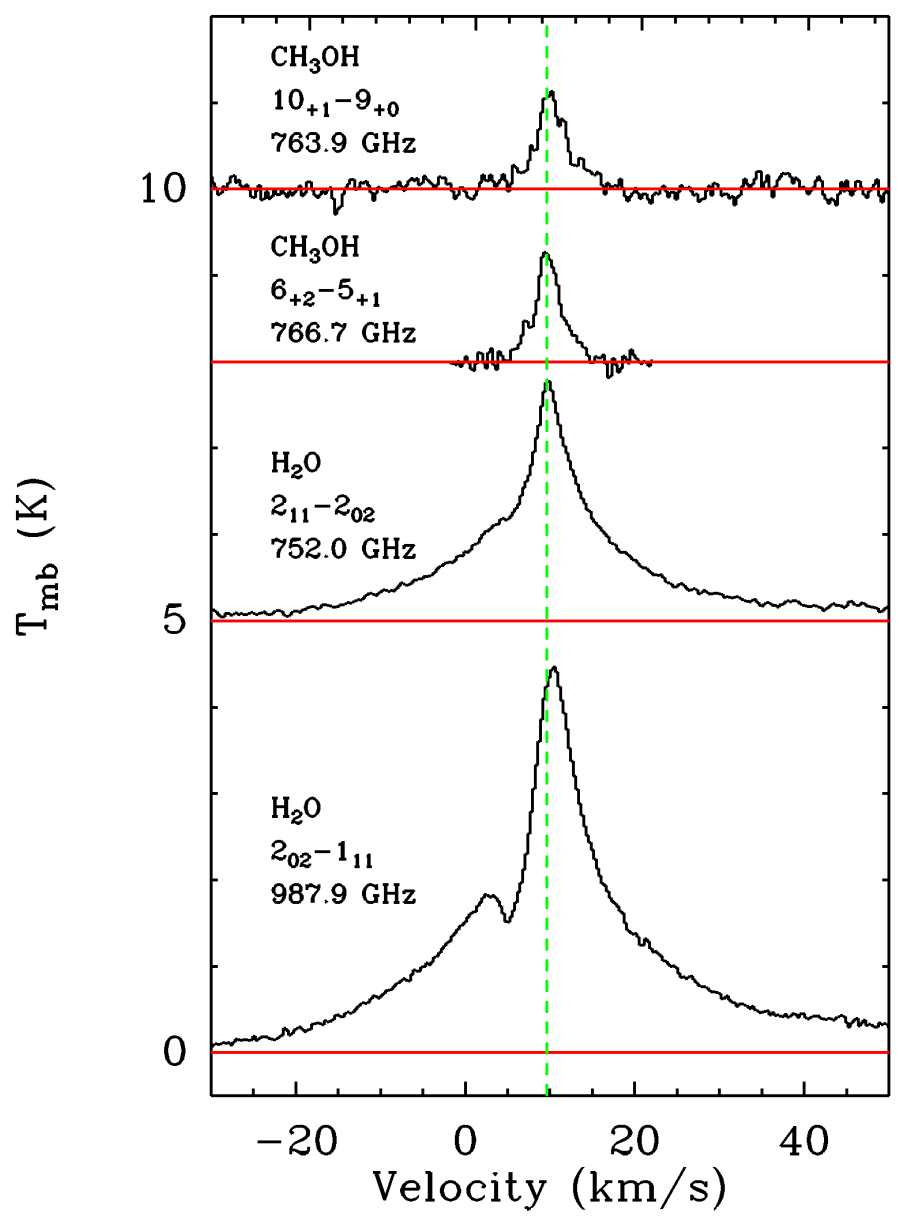

Fig. 4. HIFI Line profiles of the $\mathrm{CH}_{3} \mathrm{OH} 10_{+1}-9_{+0}(763.9 \mathrm{GHz})$ and $6+2-5_{+1}(766.7 \mathrm{GHz})$ and $\mathrm{H}_{2} \mathrm{O} 2_{11}-2_{02}(752.0 \mathrm{GHz})$ and $22_{02}-1_{11}$ $(987.9 \mathrm{GHz})$ transitions. The $\mathrm{CH}_{3} \mathrm{OH}$ lines are multiplied by a factor of 5. Spectra are binned to $0.3 \mathrm{~km} \mathrm{~s}^{-1}$ bins. Respective baselines are shown in red. The $V_{\mathrm{LSR}}$ of NGC 2071 is shown in green. We note that the wings of the $\mathrm{H}_{2} \mathrm{O}$ lines extent beyond the horizontal scale.

at all three positions for all transitions. Only the $\mathrm{CH}_{3} \mathrm{OH}$ transition at $218.44 \mathrm{GHz}$ hints at resolved emission at " $\mathrm{F}$ ", albeit only marginally ( $\sim 1.2 \times$ the beam). All $\mathrm{CH}_{3} \mathrm{CN}$ emission is unresolved at positions " $A$ " and "B" and undetected at " $F$ ". We note that SMA observations resolve out any structure larger than 13 " (van Kempen et al. 2012). The narrow $\mathrm{H}_{2} \mathrm{CO}$ component seen in Johnstone et al. (2003) between 2.2 and $2.9 \mathrm{~km} \mathrm{~s}^{-1}$, is not detected and assumed to be resolved out. It thus originates within a component that is smoothly distributed on scales of $\sim 4000 \mathrm{AU}$ or larger.

After correcting for the difference in beam size, the sum of the three regions seen in the $\mathrm{H}_{2} \mathrm{CO} 3_{03}-2_{02}$ transition reproduces $96 \%$ of the emission listed in Johnstone et al. (2003). For the $\mathrm{H}_{2} \mathrm{CO} 3_{22}-2_{21}$ transition, the $16 \mathrm{~km} \mathrm{~s}^{-1}$ wide component is not detected by the SMA. The sum of the three regions equals to $75 \%$ of the total intensity reported in Johnstone et al. (2003). It should be noted that the wide velocity component seen in Johnstone et al. (2003) was solely detected in the $\mathrm{H}_{2} \mathrm{CO} 3_{22}-2_{21}$ transition, and not in any other line included in their study. For the $4_{2}-3_{1}$ line at $218.44 \mathrm{GHz}$, the three regions reproduce $91 \%$ of the observed JCMT emission. Given the uncertainties in absolute flux calibration of both the JCMT (20\%) and SMA (15\%), organic emission seen with the JCMT is almost fully recovered by the SMA by summing the emission of the three regions. We thus assume that for all lines detected by the SMA, emission is 

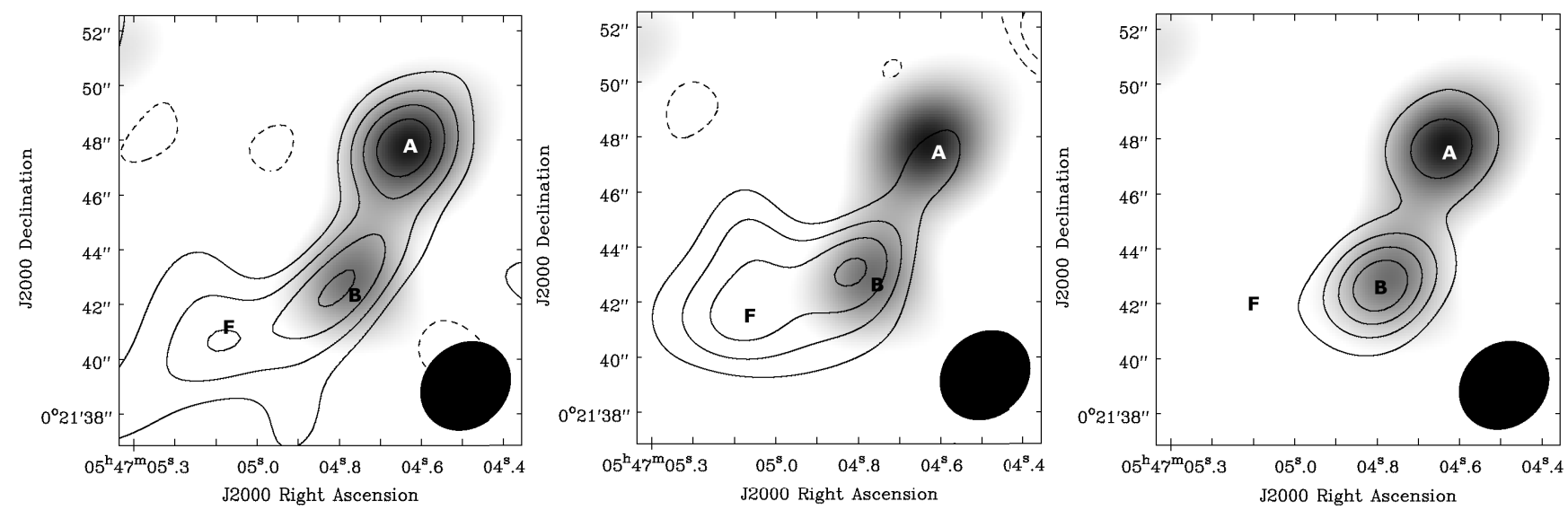

Fig. 5. $\mathrm{H}_{2} \mathrm{CO} 218.48 \mathrm{GHz}$ (left), $\mathrm{CH}_{3} \mathrm{OH} 218.44 \mathrm{GHz}$ (middle) and $\mathrm{CH}_{3} \mathrm{CN} 220.71 \mathrm{GHz}$ (right) maps, revealing that the emission originates from the "A" and "B" cores and the blue flow (marked A, B and F). Contours are overplotted on the continuum emission from van Kempen et al. (2012) in $20,40,60$ and $80 \%$ of the peak line integrated intensity, which is always located at core B.

Table 3. Detected $\mathrm{CH}_{3} \mathrm{OH}, \mathrm{CH}_{3} \mathrm{CN}$ and $\mathrm{H}_{2} \mathrm{CO}$ with the SMA.

\begin{tabular}{|c|c|c|c|c|c|c|c|}
\hline Molecule & Transition & Type & $\begin{array}{l}\text { Frequency } \\
{[\mathrm{GHz}]}\end{array}$ & $\begin{array}{l}E_{\text {up }} \\
{[\mathrm{K}]}\end{array}$ & \multicolumn{3}{|c|}{$\begin{array}{l}\mathrm{A} \\
{\left[\mathrm{K} \mathrm{km} \mathrm{s}^{-1}\right]}\end{array}$} \\
\hline \multirow[t]{6}{*}{$\mathrm{CH}_{3} \mathrm{OH}$} & $3-2-4-1$ & E & 230.03 & 39.8 & 3.7 & 12.7 & 5.4 \\
\hline & $4_{+2}-3_{+1}$ & $\mathrm{E}$ & 218.44 & 45.5 & 5.7 & 37.1 & 50.5 \\
\hline & $8_{-1}-7_{0}$ & $\mathrm{E}$ & 229.76 & 89.1 & 48.4 & 64.2 & 76.7 \\
\hline & $8_{+0}-7_{+1}$ & $\mathrm{E}$ & 220.08 & 96.6 & 4.6 & 24.2 & 14.9 \\
\hline & $10_{+2}-9_{+3}$ & A & 231.28 & 110.9 & 8.2 & 14.0 & - \\
\hline & $10_{+2}-9+3$ & A & 231.42 & 110.9 & 19.4 & 11.3 & 8.5 \\
\hline \multirow{3}{*}{$\mathrm{H}_{2} \mathrm{CO}$} & $3_{0,3}-2_{0,2}$ & para & 218.22 & 21.0 & 213 & 267.0 & 184.0 \\
\hline & $3_{2,2}-2_{2,1}$ & para & 218.48 & 68.1 & 90.8 & 108.8 & 56.4 \\
\hline & $3_{2,1}-2_{2,0}$ & para & 218.76 & 68.1 & 93.1 & 103.0 & 53.9 \\
\hline \multirow[t]{2}{*}{$\mathrm{CH}_{3} \mathrm{CN}$} & $0 / 1$ & & 220.75 & 68.9 & 48.3 & 62.4 & - \\
\hline & 2 & & 220.73 & 97.4 & 45.9 & 72.6 & - \\
\hline$J=$ & 3 & & 220.71 & 133.2 & 26.0 & 34.2 & - \\
\hline $12-11$ & 4 & & 220.68 & 183.1 & 20.1 & 21.7 & - \\
\hline \multirow[t]{2}{*}{ ladder } & 5 & & 220.64 & 247.4 & - & 14.1 & - \\
\hline & 6 & & 220.59 & 325.9 & - & 13.2 & - \\
\hline
\end{tabular}

Notes. Lines are integrated over their width and converted to Kelvin $\mathrm{km} \mathrm{s}^{-1}$. Uncertainty of the flux density on all lines is $15 \%$.

produced in these three regions and not in any undetected component resolved out, or other regions within the JCMT beam.

\section{Analysis}

\subsection{Excitation of $\mathrm{CH}_{3} \mathrm{OH}$ and $\mathrm{H}_{2} \mathrm{CO}$}

Most $\mathrm{CH}_{3} \mathrm{OH}$ and $\mathrm{CH}_{3} \mathrm{CN}$ transitions inherently possess high critical densities $\left(10^{9} \mathrm{~cm}^{-3}\right.$ or higher, see Johnstone et al. 2003). As such, excitation conditions can reliably be derived using the one-dimensional non-LTE radiative transfer code RADEX (van der Tak et al. 2007) from line ratios. The transitions are assumed to be completely optically thin and fill the beam, following Kama et al. (2010). Optical depth effects and other radiative transfer effects such as IR pumping are discussed later.

A large grid of RADEX models was run to derive E-type $\mathrm{CH}_{3} \mathrm{OH}, \mathrm{H}_{2} \mathrm{CO}$ and $\mathrm{CH}_{3} \mathrm{CN}$ emission line predictions between 215 and $1115 \mathrm{GHz}$, using datafiles provided by LAMDA $^{5}$

\footnotetext{
5 The Leiden Atomic and Molecular DAtabase: See http://www . strw. leidenuniv.nl/ moldata/
}

(Schöier et al. 2005) with the upward rate coefficients calculated at the appropriate temperature. Temperatures ranged from 50 to 500 Kelvin, while densities were varied between $10^{4} \mathrm{~cm}^{-3}$ and $10^{9} \mathrm{~cm}^{-3}$.

Most collisional rate coefficient are limited to $J_{\text {up }}=15$ and temperatures of $200 \mathrm{~K}$. At higher kinetic temperatures, downward collisional rate coefficients of $200 \mathrm{~K}$ are used. The RADEX line width was set to $6 \mathrm{~km} \mathrm{~s}^{-1}$. A column density of only $10^{7} \mathrm{~cm}^{-2}$ was chosen, since we are interested in line ratios of optically thin transitions. Differential beam dilution is corrected using the method described in the Appendix of B of Tafalla et al. (2010) assuming the regions are point sources. Dilution factors are thus proportional to $\theta_{\mathrm{MB}}^{-2}$. However, test using the onedimensional source structure $\left(\theta_{\mathrm{MB}}^{-1}\right)$ show little to no changes.

Predictions of line ratios were compared to the observed ratios of the five E-type $\mathrm{CH}_{3} \mathrm{OH}$ lines detected with the SMA for each region. Figure 6 shows the results of a Pearson's $\chi^{2}$ test $^{6}$. The errors on individual ratios are $21 \%$. The ratios reveal clear differences in the physical conditions between the regions, presented in Fig. 6. The best fit found for region " $F$ " is a density of $4 \pm 0.1 \times 10^{7} \mathrm{~cm}^{-3}$. Region "A" is constrained to a density of $2 \pm 0.8 \times 10^{7} \mathrm{~cm}^{-3}$, while region " $\mathrm{B}$ " is constrained to densities $>2 \times 10^{8} \mathrm{~cm}^{-3}$. Temperatures are loosely constrained to values $>100$ Kelvin.

Better constraints on the kinetic temperature can be obtained through comparison of the line ratio of $\mathrm{H}_{2} \mathrm{CO} 3_{0,3}-2_{0,2}$ over $3_{2,2}-$ $2_{2,1}$ with the RADEX model. Figure 7 shows the resulting line ratios of our RADEX grid of these two $\mathrm{H}_{2} \mathrm{CO}$ lines. The observed ratios of the three regions are plotted at the densities derived using the $\chi^{2}$ tests of the $\mathrm{CH}_{3} \mathrm{OH}$ ratios. This results in temperature constraints of $215_{-70}^{+200} \mathrm{~K}$ for region "A", $150_{-50}^{+280} \mathrm{~K}$ for region " $\mathrm{B}$ " and $100_{-15}^{+40} \mathrm{~K}$ for region " $\mathrm{F}$ ". The error bar on the temperature can be significant, especially for regions " $A$ " and "B". Much higher temperatures $(>400 \mathrm{~K})$ are within uncertainty. This is in part due to the lack of collision rate coefficients above $300 \mathrm{~K}$ for $\mathrm{H}_{2} \mathrm{CO}$. However, constraints on lower temperatures are much better. Even with these large error, all three regions are warmer than the surrounding envelope, but not hot $(T>500 \mathrm{~K})$. Owing to the molecular structure of $\mathrm{H}_{2} \mathrm{CO}$, there is no difference between using the $3_{2,2}-2_{2,1}$ or $3_{2,1}-2_{2,0}$ transition. Given the near

6 Pearson's test is defined as $\left.\chi^{2}=\sum_{i=1}^{n} \frac{\left(R_{i}-P_{i}\right)^{2}}{P_{i}}\right)$ with $R_{i}$ the observed ratio and $P_{i}$ the calculated ratio over a total of $n$ ratios, in which the smallest $\chi^{2}$ gives the best fit. 

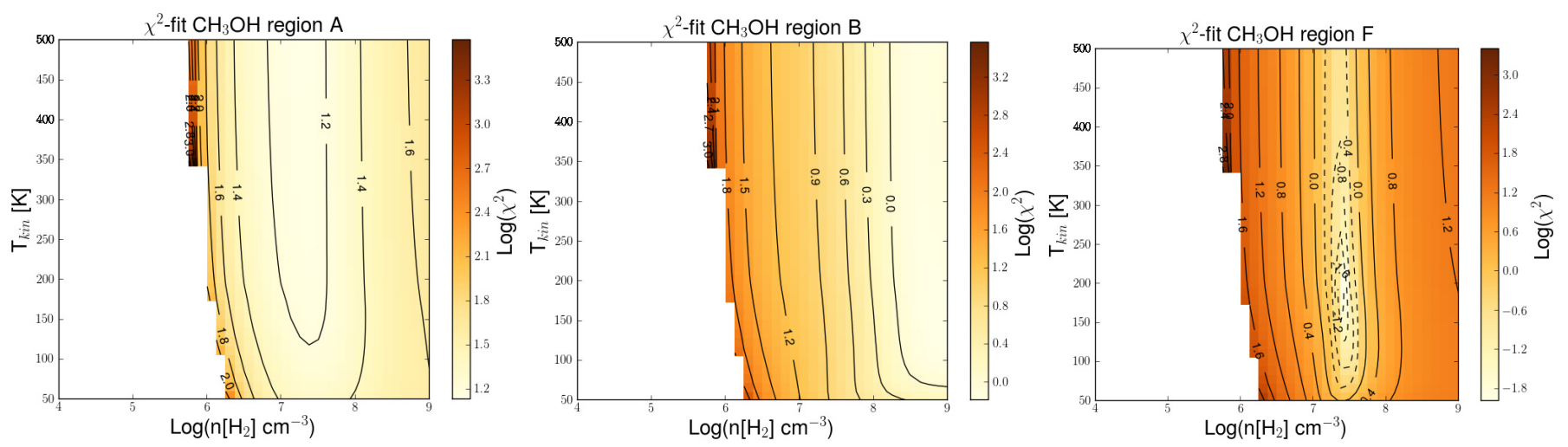

Fig. 6. Pearsons $\chi^{2}$ results of line ratios predicted by RADEX modeling and the observed ratio for all detected $\mathrm{E}_{\text {-type }} \mathrm{CH}_{3} \mathrm{OH}$ lines of the three regions (left: A, middle: $\mathrm{B}$, right: F). The color-scales are chosen such that lighter color indicates lower $\chi^{2}$ values and better agreement with observed line ratio and models. Densities are well fitted with values of $2 \times 10^{7} \mathrm{~cm}^{-3}$ for region " $\mathrm{A}$ ", $>3 \times 10^{8} \mathrm{~cm}^{-3}$ for region "B" and $4 \times 10^{7} \mathrm{~cm}^{-3}$ for region "F",

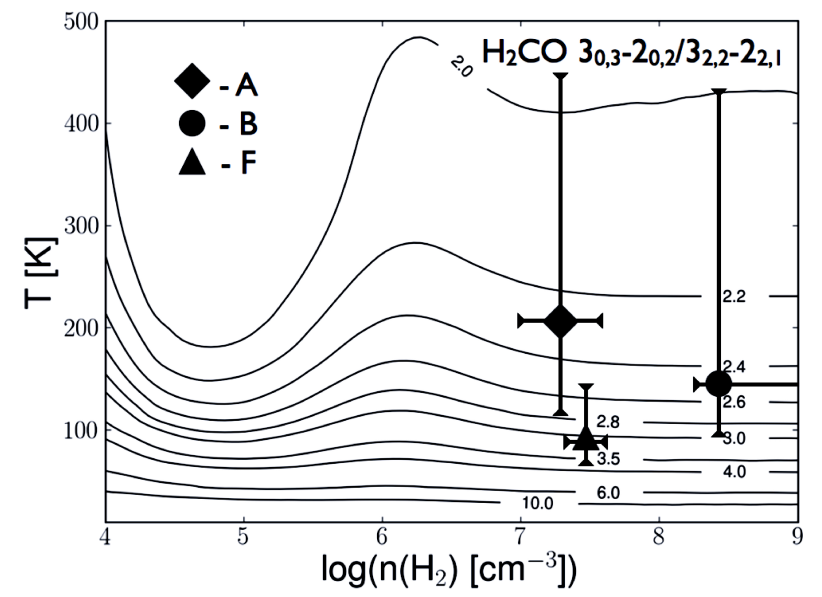

Fig. 7. Line ratio of $\mathrm{H}_{2} \mathrm{CO} \quad 3_{0,3}-2_{0,2}$ over $3_{2,2}-2_{2,1}$ modeled with RADEX. Observed line ratios are shown with a diamond (A), circle (B) and triangle (F) at the best-fit densities derived using the $\mathrm{CH}_{3} \mathrm{OH}$ line ratios (see text). Error bars for the uncertainties originate from the line ratio error $(21 \%)$. Errors on the densities originate from the $\chi^{2}$ fitting. Temperatures are $215 \mathrm{~K}(\mathrm{~A}), 150 \mathrm{~K}(\mathrm{~B})$ and $100 \mathrm{~K}(\mathrm{~F})$.

identical line ratios of either with respect to the $3_{0,3}-2_{0,2}$ line, the temperature constraint can be assumed to be robust and not affected by observational biases.

The narrow range of constraints for " $F$ " imply that the emission in that region comes from a more homogeneous medium than "A" or "B". The less restrictive fits in density around the protostellar positions "A" and "B" likely indicate density gradients within the emitting region. The significant differences in density $\left(2 \times 10^{7} \mathrm{~cm}^{-3}\right.$ versus $\left.3 \times 10^{8} \mathrm{~cm}^{-3}\right)$ and, to a lesser extent, temperature $(215 \mathrm{~K}$ versus $150 \mathrm{~K})$ between " $\mathrm{A}$ " and " $\mathrm{B}$ " are surprising given the lack of difference in line width (6 $\mathrm{km} \mathrm{s}^{-1}$ for both), individual line emission of the $\mathrm{H}_{2} \mathrm{CO}$ $3_{2,2}-2_{2,1}$ line (109 versus $91 \mathrm{~K} \mathrm{~km} \mathrm{~s}^{-1}$ ), $3_{0,3}-2_{0,2}$ line (267 vs. $\left.213 \mathrm{~K} \mathrm{~km} \mathrm{~s}^{-1}\right)$, resolved dust emission $\left(0.13 \mathrm{Jy} \mathrm{beam}^{-1}\right.$ versus $0.12 \mathrm{Jy} \mathrm{beam}^{-1}$, see Table 3 and Fig. 2 in van Kempen et al. 2012) or stellar $\left(0.9 M_{\odot}\right.$ versus $\left.0.5 M_{\odot}\right)$ and individual envelope masses $\left(8.2 M_{\odot}\right.$ versus $\left.14.2 M_{\odot}\right)$. The main difference between the two regions is that "B" powers the large NGC 2071 outflow, while "A" possesses a much weaker flow (Carrasco-González et al. 2012).

\subsection{Optical depth}

The assumption that the observed methanol lines are all optically thin must be investigated further. Using RADEX one can determine the column densities and in turn the associated optical depths. It should be noted that the solution is degenerate with an assumed source size within a single beam. Smaller source sizes require higher column densities and thus higher optical depths are to reproduce the same amount of emission. If the source fills the SMA beam, as assumed above, a column of $4 \times 10^{15} \mathrm{~cm}^{-2}$ was derived to best fit the observed flux densities. In this model, optical depths are typically 0.01 or lower. RADEX shows that to produce optically thick lines with the observed emission, a column of $8 \times 10^{17} \mathrm{~cm}^{-2}$ or higher is required. Such a column corresponds to a source size 14 times smaller than the SMA beam, equal to $\sim 90 \mathrm{AU}$.

However, at these high column densities, some lines will mase, affecting line ratios. The $218.44 \mathrm{GHz}$ line is a weak maser, but shows similar line strengths when masing. The largest effect is seen for the ratios between the 218.44, 220.08 and $229.76 \mathrm{GHz}$ lines. At higher columns, RADEX shows the 229.76 line as an absorption feature of similar depth as the emission of the $218.44 \mathrm{GHz}$ line. However, no absorption was seen. In addition, the 220.08 line should be 100 times brighter than the $218.44 \mathrm{GHz}$ line, while a ratio of about 0.5 to 2.5 was observed. From this, we can thus exclude that the emission originates from a very small region with a very high column density. The theoretically largest column that correctly reproduces the observed line ratios is a few times $10^{16} \mathrm{~cm}^{-2}$, corresponding to an optical depth $(\tau)$ of 0.4 . The assumption that all $\mathrm{CH}_{3} \mathrm{OH}$ emission is optically thin is thus justified.

Besides a high optical depth, IR pumping of low density $\left(10^{3} \mathrm{~cm}^{-3}\right)$ methanol gas could also influence the observed line ratios for $v=0$ rotationally excited transitions (Leurini et al. 2007). Observations of torsionally excited methanol lines $(v=1)$ are typically invoked to break the degeneracy. Although over 6 torsionally excited transitions were covered in the bandpass of the SMA, none were detected for the achieved sensitivity of (71 $\mathrm{mJy}$ in $2 \mathrm{~km} \mathrm{~s}^{-1}$ bins). In addition, no $v=1$ transitions were detected in the HIFI bandpass. This indicates IR pumping has little to no effect on the methanol emission. RADEX experiments with a gray body radiation field and lower densities showed that although the ratios of non-masing lines are indeed reproduced, it is impossible to simultaneously quench all three maser lines and produce the correct ratios. It is possible to add a very small ( $<50 \mathrm{AU})$ IR pumped component to the observed ratios. This 
can account for $25 \%$ of the observed line strengths. Whether or not such a component is present requires deep observations of the $v=1$ transitions, e.g., using the band around $241.2 \mathrm{GHz}$.

\subsection{Excitation of $\mathrm{CH}_{3} \mathrm{CN}$}

RADEX results for $\mathrm{CH}_{3} \mathrm{CN}$ line ratios cannot improve on the solutions for the physical conditions obtained with $\mathrm{H}_{2} \mathrm{CO}$ and $\mathrm{CH}_{3} \mathrm{OH}$, as all detected lines are part of the $J=12-11$ ladder. From the RADEX models we derive a kinetic temperature of $200 \mathrm{~K}$ or higher for " $\mathrm{A}$ " and $\sim 150 \mathrm{~K}$ for " $\mathrm{B}$ ". These values are consistent with the results of $\mathrm{CH}_{3} \mathrm{OH}$ and $\mathrm{H}_{2} \mathrm{CO}$. It is thus reasonable to assume that the $\mathrm{CH}_{3} \mathrm{CN}$ emission originates from the same gas as $\mathrm{CH}_{3} \mathrm{OH}$ and $\mathrm{H}_{2} \mathrm{CO}$ in " $\mathrm{A}$ " and " $\mathrm{B}$ ". However, $\mathrm{CH}_{3} \mathrm{CN}$ emission is absent from " $\mathrm{F}$ ".

With the optically thin assumption, the similarity of line ratios between $\mathrm{CH}_{3} \mathrm{CN}, \mathrm{H}_{2} \mathrm{CO}$ and $\mathrm{CH}_{3} \mathrm{OH}$ in " $\mathrm{A}$ " and "B", and the observed strength of the emission of $\mathrm{H}_{2} \mathrm{CO}$ and $\mathrm{CH}_{3} \mathrm{OH}$ in " $F$ ", the relative abundance of $\mathrm{CH}_{3} \mathrm{CN}$ with respect to $\mathrm{H}_{2} \mathrm{CO}$ and $\mathrm{CH}_{3} \mathrm{OH}$ must be almost two orders of magnitude lower at " $F$ " then in "A" and "B". Excitation conditions cannot explain the lack of $\mathrm{CH}_{3} \mathrm{CN}$ emission at "F".

\subsection{Velocity profile: comparison to $\mathrm{H}_{2} \mathrm{O}$}

The behavior of the observed line ratio between $\mathrm{CH}_{3} \mathrm{OH}$ and $\mathrm{H}_{2} \mathrm{O}$ emission lines away from the source velocity differentiates between two potential formation routes of $\mathrm{H}_{2} \mathrm{O}$ (Suutarinen et al. 2014). If gas-phase $\mathrm{CH}_{3} \mathrm{OH}$ and $\mathrm{H}_{2} \mathrm{O}$ are created solely through grain mantle evaporation, the ratio will be flat. However, if gasphase synthesis of $\mathrm{H}_{2} \mathrm{O}$ is taking place, the ratio will drop as more $\mathrm{H}_{2} \mathrm{O}$ is created from shocked material. A significant optical depth in the water line causes observed ratios to be higher than physical processes would produce. Although an optical depth cannot be derived because of a lack of observed isotopologues, it is hypothesized that the $\mathrm{H}_{2} \mathrm{O} 752 \mathrm{GHz}$ may be affected near the line center (between 8.3 to $10.3 \mathrm{~km} \mathrm{~s}^{-1}$ ). In outflows of lowmass protostars (e.g., Kristensen et al. 2010), optical depths are factors of a few at line center.

However, owing to the high critical density of water transitions $\left(>10^{8} \mathrm{~cm}^{-3}\right.$ for both the 987 and $752 \mathrm{GHz}$ line), the assumed optically thick emission is most often effectively thin. The effects of water opacity on the ratio are still relatively small. See, e.g., Fig. 3 of Suutarinen et al. (2014). For velocities where optical depth may play a role, the ratio is overestimated by a factor of 2 at most.

Figure 8 shows the ratio of $76310_{1}-9_{0} \mathrm{CH}_{3} \mathrm{OH}$ transition and the $7522_{11}-2_{02} \mathrm{H}_{2} \mathrm{O}$ line. The observed $\mathrm{CH}_{3} \mathrm{OH} / \mathrm{H}_{2} \mathrm{O}$ ratio clearly drops as a function of velocity on the blue wing. The red wing also is falling, although the number of channels not affected by optical depth is marginal. Velocities between 8.5 and $10.2 \mathrm{~km} \mathrm{~s}^{-1}$ are thought to be affected by optical depth. As seen

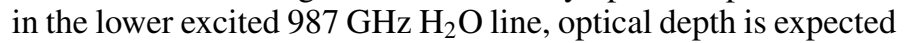
to be at its highest there causing the ratios at these velocities to be higher.

Line ratios of other $\mathrm{CH}_{3} \mathrm{OH} / \mathrm{H}_{2} \mathrm{O}$ combinations (e.g., the group of methanol lines at $766 \mathrm{GHz}$ ) show near identical profiles: A clear drop in the ratio on the blue side in combination with a drop on the red side and a middle likely affected by optical depth. Ratios derived using the $987 \mathrm{GHz} \mathrm{H}_{2} \mathrm{O}$ line are more affected by optical depth, but still reproduce the falling ratio of the blue wing.

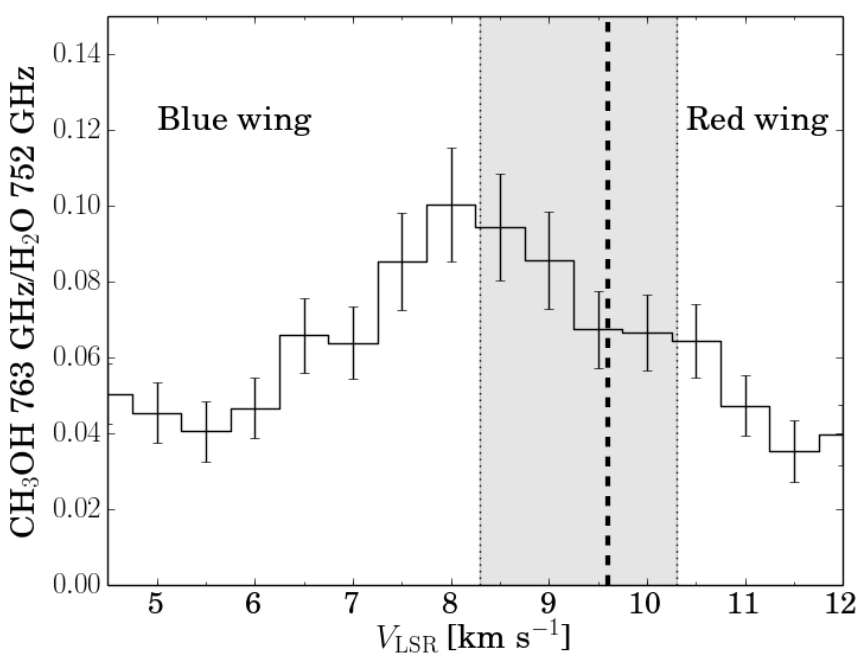

Fig. 8. Line ratio of the $\mathrm{CH}_{3} \mathrm{OH} 10_{+1}-9_{+0}$ over the $\mathrm{H}_{2} \mathrm{O} 2_{11}-2_{02}$ transitions, both rebinned to $0.5 \mathrm{~km} \mathrm{~s}^{-1}$. The $V_{\mathrm{LSR}}$ is shown with a dashed line. The range of velocities where the high optical depth of the water line is hypothesized to affect the derived ratio is shown in gray. No correction has been applied (see text).

\section{Discussion: Origin of organic emission}

Using the optically thin approximation, the observed spatial separation between the regions and lack of large-scale $\mathrm{CH}_{3} \mathrm{OH}$ emission, $\mathrm{CH}_{3} \mathrm{OH}$ HIFI detection originate solely in the three regions. The detected flux density for each line is a simple sum,

$\mathrm{CH}_{3} \mathrm{OH}=C *\left(X_{\mathrm{A}} * R_{\mathrm{A}}+X_{\mathrm{B}} * R_{\mathrm{B}}+X_{\mathrm{F}} * R_{\mathrm{F}}\right)$,

where $\mathrm{CH}_{3} \mathrm{OH}$ is the total observed emission within the HIFI beam, $R_{X}$ the predicted emission from RADEX of region " $\mathrm{A}$ ", "B", or " $F$ " for that transition, $\mathrm{X}$ the relative contribution and $\mathrm{C}$ a constant to ensure the sum equals observed intensities.

If it is assumed $X_{\mathrm{A}}, X_{\mathrm{B}}$, and $X_{\mathrm{F}}$ do not vary for individual $\mathrm{CH}_{3} \mathrm{OH}$ lines, and are thus not vectors themselves, the set of equations can be solved by rewriting the equation for individual $\mathrm{CH}_{3} \mathrm{OH}$ line contributions into a vector notation covering all the HIFI $\mathrm{CH}_{3} \mathrm{OH}$ lines,

$\boldsymbol{C H}_{3} \mathrm{OH}=\boldsymbol{C} *\left(X_{\mathrm{A}} * \boldsymbol{R}_{\mathrm{A}}+X_{\mathrm{B}} * \boldsymbol{R}_{\mathrm{B}}+X_{\mathrm{F}} * \boldsymbol{R}_{\mathrm{F}}\right)$,

where $\mathrm{CH}_{3} \mathrm{OH}$ is a vector, one column wide, containing the HIFI-detected emission line flux densities, and $\boldsymbol{R}_{\boldsymbol{X}}$ are the vectors, again one column wide, containing the predicted fluxes obtained from RADEX for the 14 HIFI E-type methanol transitions with $J \leq 15$. Higher $J$ transitions are not available in the molecular datafile. A-type methanol is not included, since no constraints from the SMA are given for A-type methanol transitions. Even assuming they originate under identical conditions with an abundance ratio of $1: 1$, inclusion of A-type methanol does not contribute to the analysis below. $\boldsymbol{C}$ is the vector containing the constant $C$ for each transition. Under the assumption that the source size is the same for all transitions, $\boldsymbol{C}$ will depend on the model parameters. Its only dependency is on the frequency of the transition. This known quantity determines the beam dilution in the HIFI beam. We realize that Eq. (2) is an oversimplification, as $X_{\mathrm{A}}, X_{\mathrm{B}}$, and $X_{\mathrm{F}}$ may vary from transition to transition. Similarly, source sizes may become smaller for higher excited transitions. However, given the uncertainties in the observed emission, these simplifications produce acceptable results.

Solving Eq. (2) using a least squares method, optimal values of $13 \%$ for $X_{\mathrm{A}}, 71 \%$ for $X_{\mathrm{B}}$ and $16 \%$ for $X_{\mathrm{F}}$ are derived. 
With constant calibration uncertainties (in the case for Herschel a conservative value of $10 \%$ on the flux densities is assumed), the square of the error on the relative contributions equals the mean error times the rank of the matrix divided by the number of transitions ${ }^{7}$. The solution actually has a rank of only 2 (as opposed to 3 as was expected). The reason for this reduction in rank is that emission contributions in the conditions for " $F$ " and "B" are very similar for the transitions probed. These produce similar ladders and none of the transitions used can distinguish them. The statistical error on the values of 13,71 and $16 \%$ is $4 \%$.

For the detected SMA transitions, the mean of the individual relative contributions is indeed close to this value. Relative contributions of some of the individual transtions also adhere to the relation. For example, the $\mathrm{CH}_{3} \mathrm{OH} 3-2-4-1$ line in Table 3, "A" contributes $17 \%$, "B" contributes $58 \%$ and " $\mathrm{F}$ " contributes $25 \%$. However, clear variations are visible. Stronger contributions from "F" are detected for some transitions. Whether or not these are introduced by optical depths effects because of the beam at "B", or if the inferred density gradient at "B" comes into play, remains to be seen.

Tests using other density and temperature parameters for regions " $A$ " and "B" (i.e., higher temperatures and densities) also show variations at the level of $5 \%$ in the inferred contributions.

Deviations in relative contributions for higher excitation lines were subsequently investigated. Changes larger than $20 \%$ in relative contributions to higher excitation lines (e.g., by forcing " $\mathrm{A}$ " to contribute $50 \%$ of the observed emission of a $\mathrm{CH}_{3} \mathrm{OH}$ line with $E_{\text {up }}=400 \mathrm{~K}$ ) become very problematic. In such cases, HIFI detections with $E_{\text {up }}<250 \mathrm{~K}$ significantly violate the constraints set by the SMA detections. Variations of $5-10 \%$ are likely from transition to transition. In addition, emission from "B" and " $F$ " cannot be disentangled from Herschel transitions alone. The percentages of " $B$ " and " $F$ " originate from the best solution in the least squares fitting and the mean of the SMA emission quantities.

The absence of molecular data beyond $J=15$ likely hinders further interpretation. To improve on this analysis, a full radiative transfer model with power law exponents describing envelope structures is warranted. This is considered to be beyond the scope of this paper.

In the end, the emission contribution from each region to all HIFI detections are estimated to be:

$$
\begin{aligned}
& -15-20 \% \text { originates in region "A" } \\
& -60-70 \% \text { in region "B" } \\
& -15-25 \% \text { in region " } F " .
\end{aligned}
$$

In region "F", it is clear that the emission is related to the outflowing gas in position, but not in velocity. The absence of any stellar source rules out any possibility of disk-related scenarios or a hot corino. Whether or not the emission from "A" and "B" are related to the outflow or are part of a hot corino is debatable. However, a case in which the outflow plays a strong role is more likely.

Typical spherical envelope models with power-law density profiles show the derived higher densities of a few times $10^{7} \mathrm{~cm}^{-3}$ to a few times $10^{8} \mathrm{~cm}^{-3}$ to be located on scales of $\simeq 100$ AU. Gas temperatures of $150-200 \mathrm{~K}$ are located at scales of $50 \mathrm{AU}$ or less, very close to the inner edge of the model

\footnotetext{
7 The error $\sigma$ is calculated from $\sigma^{2}=\sigma_{f}^{2} * M / N$ with $\sigma_{f}$ the mean flux density error, $M$ the rank of the matrix and $N$ the number of entries.
}

(Shirley et al. 2002; Jørgensen et al. 2002; Kristensen et al. 2012). Envelopes around the individual NGC 2071 sources have been found to be very similar to these typical low-mass protostellar envelopes (van Kempen et al. 2012).

A scenario in which organics formed within the icy grain mantles, were subsequently liberated by either sputtering or grain-grain collisions and are currently emitting in a compressed, UV heated gas component is much more likely. Recently, Kristensen et al. (2013) found kinematic components in $\mathrm{H}_{2} \mathrm{O}$ resolved lines consistent with dissociative jet shocks in the inner $100 \mathrm{AU}$ to explain distinct velocity components in $\mathrm{H}_{2} \mathrm{O}$ spectra. Such components produce emission offset from the central velocity and as such cannot originate in a hot-core like environment. They are too powerful to be associated with potential non-dissociative shocks along the outflow cavity wall. An irradiated shock was inferred from the low abundances in that component.

Outflows are known to be present at the smallest scales: " $\mathrm{B}$ " is known to be the launching point of the large-scale bipolar jet of NGC 2071 (Skinner et al. 2009). "A" is a source of strong water masers. The maser spots display significant velocity shifts, indicative of jet activity (Torrelles et al. 1998; Carrasco-González et al. 2012).

\subsection{Evidence for $\mathrm{CH}_{3} \mathrm{OH}$ destruction and $\mathrm{H}_{2} \mathrm{O}$ gas-phase synthesis}

The decreasing ratio of the line profiles (see Fig. 8) shows that the chemistry in NGC 2071 is likely similar as that found for NGC 1333 IRAS 4A and 4B (Suutarinen et al. 2014). Atomic hydrogen formed in the shock is able to destroy $\mathrm{CH}_{3} \mathrm{OH}$ through chemical reactions featuring hydrogen extraction, e.g., $\mathrm{H}+$ $\mathrm{CH}_{3} \mathrm{OH} \rightarrow \mathrm{H}_{2}+\mathrm{CH}_{2} \mathrm{OH}$ or $\mathrm{H}+\mathrm{CH}_{3} \mathrm{OH} \rightarrow \mathrm{H}_{2}+\mathrm{CH}_{3} \mathrm{O}$ (Baulch 2005). Both products are also quickly destroyed. All these reactions have activation energies of $\sim 800 \mathrm{~K}$, much lower than similar reactions capable of destroying $\mathrm{H}_{2} \mathrm{O}$ (activation energy of $\sim 10^{4} \mathrm{~K}$ ). Gas-phase synthesis of $\mathrm{H}_{2} \mathrm{O}$ apparently can take place along the entire flow, given the brightness of the lines. Methanol emission is constrained to regions " $A$ ", " $B$ " and " $F$ " by local physical conditions. Only regions of sufficient density $\left(10^{7} \mathrm{~cm}^{-3}\right)$ can shield methanol from the high-temperature gas and subsequent dissociation. The high spatial resolution observations (Fig. 5) show that the regions where these physical conditions occur are small ( $<1500 \mathrm{AU}$ in size). Observationally, we can conclude that the most likely location of these regions is in the neighborhood of protostars. This is not surprising considering envelope densities. However, correct conditions can also occur elsewhere, with no protostar nearby (region 'F").

\subsection{Evidence for ice processing of $\mathrm{CH}_{3} \mathrm{CN}$}

The main difference between $\mathrm{CH}_{3} \mathrm{CN}$ and $\mathrm{CH}_{3} \mathrm{OH}$ is its formation. Both form predominantly on grain surfaces, but their reactions proceed differently under cold conditions. $\mathrm{CH}_{3} \mathrm{CN}$ is mainly formed through $\mathrm{CN}$ reacting with $\mathrm{CO}$ and other carbon containing species, while $\mathrm{CH}_{3} \mathrm{OH}$ is a hydrogenation product of $\mathrm{CO}$. At low temperatures, hydrogen moves freely. However, thanks to the low mobility of $\mathrm{CN}, \mathrm{CH}_{3} \mathrm{CN}$ formation requires heating and/or irradiation. Garrod et al. (2008) show an increase in more than order of magnitude in both gas and ice abundances for $\mathrm{CH}_{3} \mathrm{CN}$ as a function of time in various warm-up scenarios, while the $\mathrm{CH}_{3} \mathrm{OH}$ or $\mathrm{H}_{2} \mathrm{CO}$ formation remains unaltered compared to the cold phase formation. Additional gas-phase formation routes for $\mathrm{CH}_{3} \mathrm{CN}$ after grain mantle evaporation cannot be 
invoked, as these typically produce abundances almost two orders of magnitude lower than the grain surface formation route (see Figs. 7 to 9 and Appendix 2 of Garrod et al. 2008).

At region "F", little to no ice processing must have taken place owing to the absence irradiation/heating at earlier stages, as opposed to the presence of a protostar at " $\mathrm{A}$ " and " $\mathrm{B}$ ". The young dynamical age of the NGC 2071 outflow $\left(<10^{4} \mathrm{yr}\right.$, van Kempen et al., in prep.) further corroborates this, limiting the production of $\mathrm{CH}_{3} \mathrm{CN}$.

\section{Conclusions}

This paper presented new data obtained with the SMA and Herschel-HIFI on the emission of organics around the cluster NGC 2071 and its outflow. The data were analyzed using a non-LTE radiative transfer code. The following conclusions are drawn:

- All organic emission detected by HIFI and the SMA observations originates in two or three regions, labeled "A", "B" and "F". "A" and "B" are the positions of the two central protostars, while " $F$ " is a shock position in the blue outflow. "F" is spatially resolved in some lower excitation lines, but does not emit in $\mathrm{CH}_{3} \mathrm{CN}$.

- Using the non-LTE radiative transfer code RADEX, physical conditions of the three individual regions were constrained using a $\chi^{2}$ analysis of the $\mathrm{H}_{2} \mathrm{CO}$ and $\mathrm{CH}_{3} \mathrm{OH}$ emission. Densities higher than $10^{7} \mathrm{~cm}^{-3}\left(10^{8} \mathrm{~cm}^{-3}\right.$ in the case of "B") and temperatures of $100 \mathrm{~K}$ or higher were derived.

- All HIFI $\mathrm{CH}_{3} \mathrm{OH}$ detected are a sum of the emission of the three regions. On average $60-70 \%$ originates from "B", $15-20 \%$ from " $A$ " and $15-25 \%$ from " $F$ ". No line originates solely from either "B" (the densest region) or "A" (the hottest region).

- The high densities $\left(>10^{7} \mathrm{~cm}^{-3}\right)$ of the regions shield the gas-phase $\mathrm{CH}_{3} \mathrm{OH}$. Everywhere else, $\mathrm{CH}_{3} \mathrm{OH}$ is destroyed through high-temperature reactions with hydrogen and/or through destructive sputtering.

- The significant difference between $\mathrm{CH}_{3} \mathrm{OH}$ and $\mathrm{H}_{2} \mathrm{O}$ line wing shapes and extent suggests $\mathrm{H}_{2} \mathrm{O}$ formation through gasphase synthesis along the flow.

- The lack of $\mathrm{CH}_{3} \mathrm{CN}$ emission at " $\mathrm{F}$ " is attributed to a difference in ice processing history.

Small-scale structure of organic emission is of vital importance in determining its origin and thus understand chemical processes during the early stages of star formations. Future observations with ALMA such as done in Jørgensen et al. (2013) are needed to determine if all organics emission lines originate from a shockinduced scenario or a hot corino. In the case of NGC 2071, the complexity of the chemistry could not be understood by singledish observations alone.

Acknowledgements. The research of Tim van Kempen is made possible by the Allegro ARC node. Michel Fich, Carolyn $\mathrm{M}^{\mathrm{c}} \mathrm{Coey}$ and Sam Tisi are supported in this work by a Discovery Grant from NSERC and a Space Science Enhancement Program grant from the Canadian Space Agency. Doug Johnstone is supported by the National Research Council of Canada and by a Natural Sciences and Engineering Research Council of Canada (NSERC) Discovery Grant. We thank
Mihkel Kama for useful discussions on methanol detections using HIFI. Michiel Hogerheijde and Floris van der Tak are thanked for assistance on RADEX. The WISH team, in particular Silvia Leurini, is thanked for usefull discussions.

\section{References}

Baulch, D. L. 2005, J. Phys. Chem. Ref. Data, 34, 757

Bisschop, S. E., Jørgensen, J. K., Bourke, T. L., Bottinelli, S., \& van Dishoeck, E. F. 2008, A\&A, 488, 959

Bottinelli, S., Ceccarelli, C., Lefloch, B., et al. 2004a, ApJ, 615, 354

Bottinelli, S., Ceccarelli, C., Neri, R., et al. 2004b, ApJ, 617, L69

Buckle, J. V., \& Fuller, G. A. 2002, A\&A, 381, 77

Butner, H. M., Evans, N. J. I., Harvey, P. M., et al. 1990, ApJ, 364, 164

Carrasco-González, C., Osorio, M., Anglada, G., et al. 2012, ApJ, 746, 71

Caux, E., Kahane, C., Castets, A., et al. 2011, A\&A, 532, A23

Cazaux, S., Tielens, A. G. G. M., Ceccarelli, C., et al. 2003, ApJ, 593, L51

Ceccarelli, C., Caselli, P., Herbst, E., Tielens, A. G. G. M., \& Caux, E. 2007, Protostars and Planets V (Tucson: University of Arizona), 47

Chandler, C. J., Brogan, C. L., Shirley, Y. L., \& Loinard, L. 2005, ApJ, 632, 371

Comito, C., Schilke, P., Phillips, T. G., et al. 2005, ApJS, 156, 127

de Graauw, T., Helmich, F. P., Phillips, T. G., et al. 2010, A\&A, 518, L6

Flower, D. R., \& Pineau Des Forêts, G. 2010, MNRAS, 406, 1745

Garrod, R. T., Weaver, S. L. W., \& Herbst, E. 2008, ApJ, 682, 283

Johnstone, D., Boonman, A. M. S., \& van Dishoeck, E. F. 2003, A\&A, 412, 157

Jones, A. P., Tielens, A. G. G. M., \& Hollenbach, D. J. 1996, ApJ, 469, 740

Jørgensen, J. K., Schöier, F. L., \& van Dishoeck, E. F. 2002, A\&A, 389, 908

Jørgensen, J. K., Bourke, T. L., Nguyen Luong, Q., \& Takakuwa, S. 2011, A\&A, 534, A100

Jørgensen, J. K., Favre, C., Bisschop, S. E., et al. 2012, ApJ, 757, L4

Jørgensen, J. K., Visser, R., Sakai, N., et al. 2013, ApJ, 779, L22

Kama, M., Dominik, C., Maret, S., et al. 2010, A\&A, 521, L39

Kama, M., López-Sepulcre, A., Dominik, C., et al. 2013, A\&A, 556, A57

Kristensen, L. E., Visser, R., van Dishoeck, E. F., et al. 2010, A\&A, 521, L30

Kristensen, L. E., van Dishoeck, E. F., Bergin, E. A., et al. 2012, A\&A, 542, A8

Kristensen, L. E., van Dishoeck, E. F., Benz, A. O., et al. 2013, A\&A, 557, A23

Kurtz, S., Cesaroni, R., Churchwell, E., Hofner, P., \& Walmsley, C. M. 2000, Protostars and Planets IV (Tucson: University of Arizona Press), eds. V. Mannings, A. P. Boss, \& S. S. Russel, 299

Leurini, S., Schilke, P., Wyrowski, F., \& Menten, K. M. 2007, A\&A, 466, 215

López-Sepulcre, A., Taquet, V., Sánchez-Monge, Á., et al. 2013, A\&A, 556, A62

Maret, S., Ceccarelli, C., Tielens, A. G. G. M., et al. 2005, A\&A, 442, 527

Öberg, K. I., Bottinelli, S., \& van Dishoeck, E. F. 2009, A\&A, 494, L13

Ott, S. 2010, in Astronomical Data Analysis Software and Systems XIX. Proc. of a Conf. held October 4-8, 139

Pilbratt, G. L., Riedinger, J. R., Passvogel, T., et al. 2010, A\&A, 518, L1

Sandford, S. A., \& Allamandola, L. J. 1993, ApJ, 417, 815

Schöier, F. L., van der Tak, F. F. S., van Dishoeck, E. F., \& Black, J. H. 2005, A\&A, 432, 369

Scoville, N. Z., Sargent, A. I., Sanders, D. B., et al. 1986, ApJ, 303, 416

Shirley, Y. L., Evans, N. J. I., \& Rawlings, J. M. C. 2002, ApJ, 575, 337

Skinner, S. L., Sokal, K. R., Megeath, S. T., et al. 2009, ApJ, 701, 710

Spaans, M., Hogerheijde, M. R., Mundy, L. G., \& van Dishoeck, E. F. 1995, ApJ, 455, L167

Suutarinen, A. N., Kristensen, L. E., Mottram, J. C., Fraser, H. J., \& van Dishoeck, E. F. 2014, MNRAS, 440, 1844

Tafalla, M., Santiago-García, J., Hacar, A., \& Bachiller, R. 2010, A\&A, 522, A91

Takakuwa, S., Mikami, H., \& Saito, M. 1998, ApJ, 501, 501, 723

Torrelles, J. M., Gómez, J. F., Rodríguez, L. F., et al. 1998, ApJ, 505, 756

van der Tak, F. F. S., van Dishoeck, E. F., \& Caselli, P. 2000, A\&A, 361, 327

van der Tak, F. F. S., Black, J. H., Schöier, F. L., Jansen, D. J., \& van Dishoeck, E. F. 2007, A\&A, 468, 627

van Dishoeck, E. F., Blake, G. A., Jansen, D. J., \& Groesbeck, T. D. 1995, ApJ, 447, 760

van Dishoeck, E. F., Kristensen, L. E., Benz, A. O., et al. 2011, PASP, 123, 138

van Kempen, T. A., van Dishoeck, E. F., Güsten, R., et al. 2009, A\&A, 501, 633 van Kempen, T. A., Longmore, S. N., Johnstone, D., Pillai, T., \& Fuente, A. 2012, ApJ, 751, 137

Visser, R., Kristensen, L. E., Bruderer, S., et al. 2012, A\&A, 537, A55 